\title{
RE-ANALYSIS OF THE PERFORMANCE OF TRAUMATIC BRAIN INJURY PATIENTS ON THE AVAILABLE NEUROPSYCHOLOGICAL TESTS
}

\author{
${\text { Yasuhiro } \text { ITO }^{1)} \text { and Takeshi HATTA }}^{1)}$ \\ ${ }^{1)}$ Nagoya University, Japan
}

\begin{abstract}
The aim of this study was to re-analyze the performance of traumatic brain injury (TBI) patients on the available neuropsychological tests, and to introduce how to develop a desirable screening test battery. The relationship between the cognitive status and the sub-scale scores of the neuropsychological tests such as Wechsler Adult Intelligence Scale-Revised (WAIS-R) was analyzed by step-wise multipleregression analyses. Four facets of cognitive status were the criterion variables (scholastic ability, memory, attention, and information processing speed), which were rated by four medical staff members. The explanatory variables were the subtest scores of WAIS-R, Rivermead Behavioural Memory Test, Mini-Mental State Examination, Prose Recall Test, and Letter Cancellation Test. Based on the findings of multiple-regression analyses, a desirable screening test battery was proposed.
\end{abstract}

Key words: traumatic brain injury, cognitive disorder, screening test battery

In recent years, cognitive disorders of traumatic brain injury (TBI) patients have become one of the main topics in neuropsychology (e.g. Ponsford, Sloan, \& Snow, 1995; Rose \& Johnson, 1996). In this trend, researchers have begun to examine the relations between TBI and cognitive functions in more detail. For example, Whyte, Schuster, Potansky, Adams, and Coslett (2000) reported that TBI patients were markedly less attentive than demographically comparable controls. Researches also indicate that TBI patients show memory disorders. Shum, Harris, and Gorman (2000) investigated an effect of TBI on visual memory, and found that TBI patients made more false-positive errors than controls. Ewing-Cobbs, Fletcher, Levin, Iovino, and Miner (1998) found that patients showed a decline of academic achievement in a prospective longitudinal followup study ( 2 years). In their studies, TBI patients scored lower than controls on arithmetic and reading comprehension tests, and the achievement score of patients did not recover even after 6 months after injury.

In Japan, Ministry of Health, Labour and Welfare has started a new research project about cognitive disorders of TBI patients from July 2001. Based on this action, we started to investigate cognitive functions after injury. But there are several critical problems for

The authors are much indebted to Mr. Nakai, Y. (Graduate School of Human Informatics, Nagoya University), Dr. Yoshizaki, K. (Aichi Shukutoku University), Dr. Kabasawa, Y., and Mrs. Abe, J. (Nagoya City Rehabilitation Center) for their kind help to conduct this study, and to Mrs. Elizabeth Segal for her help of English editing.

Correspondence concerning this article should be addressed to Yasuhiro Ito, Department of Psychology, Graduate School of Environmental Studies, Nagoya University, Furoh-Cho, Chikusaku, Nagoya 464-8601, Japan (e-mail: s010303d@mbox.nagoya-u.ac.jp). 
evaluating cognitive functions of patients properly.

Almost all of the tests which are used in Japan were developed in Western countries. Despite the difference of culture and language between Western countries and Japan, these tests have been used without appropriate test development techniques. For example, the norms have not yet been established with large Japanese samples. Many Japanese clinicians have continued to administer the available tests without deciding which tests address which aspects of cognitive functions. As a result, content validity of these neuropsychological tests remains to be ambiguous.

Furthermore, there is no appropriate neuropsychological test with high practical validity (i.e., screening test battery). Practical validity means that a test can diagnose the cognitive disorders of TBI patients within a brief period of time. In addition to content validity, Milberg (1996) argued that practical validity is very important, with due consideration to the social background (e.g., increasing of an elderly population in the industrial countries). The comprehensive neuropsychological tests such as HalsteadReitan, Luria-Nebraska, and Luria-Christensen have been used in Western countries since 1970. However, these comprehensive tests need much time to administer because these tests consist of many sub-tests (such as memory, perception, tactile perception, language, handedness and motor, scholastic ability, and abstract reasoning; Christensen, 1975). Specialized knowledge and skill are also required for administration of these tests and evaluation of patient's performance. In the West, particularly in the United States of America, these neuropsychological tests have been used frequently because social systems for medical and neuropsychological examination are well established. These include a rigorous medical education for specialists (e.g., rehabilitation staff) and a viable payment system for medical treatment. However, in general, these tests are not used in Japanese medical routine work unless there is a particular scientific objective. One may say that Mini Mental State Examination (MMSE) is one of the desirable screening test batteries (Folstein, Folstein, \& McHugh, 1975). But MMSE may not be adequate as a screening test battery of TBI patients because it was originally developed to screen dementia in an elderly population.

One of the most urgent tasks must be to develop a new screening test battery from those available tests in the present Japanese neuropsychological situation. In order to develop a new screening test battery with content and practical validity, we re-analyzed the performance of TBI patients on the available neuropsychological tests. More concretely, we aimed to select several sub-tests among neuropsychological tests which are widely administered. While acknowledging the importance of deep-tests, we emphasize that a screening test battery is also important. Consequently, the decision to administer deep-tests can depend on the results of the screening test battery. In other words, the screening test battery can provide an efficient medical work system.

In order to achieve this, first, we tried to identify which aspects of cognitive status in TBI patients should be assessed. Abe (2000a; 2000b) investigated cognitive disorders of Japanese patients. A questionnaire survey was done 327 patients on the after effects of the injury. In this study, patients were asked about their problems in 8 cognitive facets, that is, scholastic ability, communication ability, memory, attention, planning ability, decision 
making ability, modification of failure, and information processing speed (see Prigatano, 1986). The results revealed that a large number of patients (more than $85 \%$ ) recognized their problems in 4 facets of cognitive functions; scholastic ability, memory, attention, and information processing speed. In this study, scholastic ability implied the general knowledge which is essential in daily life such as language and arithmetic. For example, most of the patients claimed impaired ability in writing and comprehension of Japanese Kanji character, mental arithmetic (e.g., 2-digits addition and multiplication), and constructing written sentences after the injury. Memory corresponds to the explicit memory for personal events or facts which were learned after the injury. The patients also complained that they were unable to remember their recent personal events and appointments (e.g., meeting with someone the following the day). Attention refers to focusing, sustaining, selective, alternating and dividing of attention (Sohlberg \& Mateer, 1989), which is similar to the central executive function of working memory (Baddeley \& Hitch, 1974; Baddeley, 2000). Most of the patients stated that, following the injury, the number of mistakes increased in daily life, and that they found it more difficult to execute two or more tasks at the same time (e.g., listening to music and reading a book). The patients claimed that the speed of psycho-motor activity (e.g., speech and writing) drastically slowed following the injury. These introspective data of Abe (2000a; 2000b) are consistent with the previous evidences (scholastic ability: Ewing-Cobbs et al., 1998; event memory: Arciniegas, Olincy, Topkoff, McRae, Cawthra, Filley, Reite, \& Adler, 2000; Cooke \& Kausler, 1995; Shum, Jamieson, Bahr, \& Wallace, 1999; Shum, Valentine, \& Cutmore, 1999; attention: Chan, 2001; Christodoulou, DeLuca, Ricker, Madigan, Bly, Lange, Kalnin, Liu, Steffener, Diamond, \& Ni, 2001; Fenwick \& Anderson, 1999; Leclercq, Couillet, Azouvi, Marliner, Martin, Strypstein, \& Rousseaux, 2000; Tate, 1999).

Based on this background, we aimed to focus on the four cognitive functions mentioned above, and tried to develop a new screening test battery. Then, we re-analyzed the performance of TBI patients on the five neuropsychological tests: Wechsler Adult Intelligence Scale-Revised (WAIS-R), Rivermead Behavioural Memory Test (RBMT), Prose Recall Test, Letter Cancellation Test, and MMSE (refer to the Method part for detailed explanation of the neuropsychological tests). The goal of this study was to identify the useful sub-tests which could estimate the four cognitive functions. For example, would the use of a sub-tests (e.g., Appointment) of RBMT be sufficient to estimate a patient's memory ability instead of the administering of the complete test?

For scholastic ability, we predicted that some verbal sub-tests of WAIS-R would be candidates of screening test battery (e.g., Vocabulary, Arithmetic, and Information) because by these sub-tests we can estimate language and computation abilities (Wechsler, 1981; Shinagawa, Kobayashi, Fujita, \& Maekawa, 1990). For memory, we hypothesized that some sub-tests of RBMT would be candidates because RBMT is constructed for estimating multiple memory functions. Coding of WAIS-R and Letter Cancellation Test would be a choice for testing attention. Prigatano (1986) argued that Coding was useful to discern attention disorder of TBI patients. Studies have also shown that Letter Cancellation Test can assess attention (e.g., Diller \& Weinberg, 1977; Lezak, 1976). For 
information processing speed, we predicted that the tests with time pressure and low memory load would be the candidates (e.g., Coding and Letter Cancellation Tests).

\section{METHOD}

\section{Participants:}

The data from 49 TBI patients ( 35 males and 14 females), who attended the Nagoya City Rehabilitation Center (NCRC) from June 1998 to April 2000, were analyzed in this retrospective study. The study was conducted with the permission of NCRC research community. The average age was 30.6 years old $(S D=11.3)$, and the median of time passed after the injury was 35 months. The median time with disturbed consciousness was 30 days. The disturbed consciousness refers to the state that patients are unable to say their name or are with disorientation. The mean of the patients' initial Glasgow Coma Scale (GCS) was 34.3 $(S D=24.9)$. GCS is a measurement of consciousness disturbance, and consciousness level is assessed by the state of eye opening, verbal response, and motor response (see Jennett \& Teasdale, 1977). Forty-four patients had been injured in a traffic accident, and 5 injured by a falling to the ground. They had no serious language problems (over 90 in Japanese Western Aphasia Battery). No patient with a premorbid history of substance dependency, intellectual disability, and neurological or psychiatric history was included in the present study.

\section{Neuropsychological Tests:}

In NCRC, many neuropsychological tests have been administered to assess cognitive functions of TBI patients in the rehabilitation routine work. However, identical test sets have not been administered to all the patients for practical reasons (e.g., insufficient time for evaluation). Therefore, we selected 5 neuropsychological tests for re-analyzing: WAIS-R, RBMT, Prose Recall Test, Letter Cancellation Test, and MMSE. These tests were administered to all 49 patients. RBMT consists of 12 sub-tests (verbal memory, visual and spatial memory, and prospective memory; see Cockburn, Wilson, Baddeley, \& Hiorns, 1990). Prose Recall Test required patients to recall a short prose (15 elements) in immediate and delayed (about 30 minutes later) conditions. The index was the number of recalled elements for each condition. In Letter Cancellation Test, the patients were asked to search five target letters (i.e., あ/a// い/i//う/u/, え/e/, お/o/) from a meaningful letter string, and to mark out them within 2 minutes as quickly and accurately as possible. The indices were the number of target letters which marked out correctly (i.e. number of hit), and the number of missed target letters per the number of targets that should be marked out (i.e. miss rate). Table 1 showed means (and $S D \mathrm{~s}$ ) on each test. The results of these tests were raw scores except for WAIS-R, of which we used the age-scaled score (Shinagawa et al., 1990).

\section{Statistical Analysis:}

First, we examined the correlations between four facets of cognitive status and the patients' performance on each neuropsychological test. Then, we clarified this relationship by step-wise multiple-regression analyses to develop a screening test battery. The criterion variables were the scores of four facets of cognitive status (i.e., scholastic ability, memory, attention, and information processing speed). These were rated by four medical staff members. These members have frequently met the patients in a course of neuropsychological assessment, rehabilitation program and/or medical examination. We instructed them to evaluate the cognitive status of patients, taking into consideration the patient's behavior in a course of rehabilitation program and medical examination, and the statements from persons close to the patient, such as family member. Because neuropsychological tests were administered by two medical staffs before this rating, we emphasized that the evaluation should not be based on the score on neuropsychological tests itself in order to minimize the rater bias. Each medical staff rated the cognitive status of each patient independently from 1 (good) to 10 (worse). The inter-rater consistency was $83.6 \%$. In case that a score for each patient differed among raters, the mean score was used. The mean subjective evaluation scores of each cognitive status was $5.64(S D=1.59)$ for scholastic ability, $5.39(S D=1.93)$ for memory, $5.11(S D=1.30)$ for attention, and $5.07(S D=1.86)$ for information processing speed. The 34 explanatory variables were the sub-test scores of WAIS-R (11), RBMT (13), and MMSE (6), Prose Recall Test (2), and Letter Cancellation Test (2). We used row scores of sub-tests as the explanatory variables except for WAIS-R, of which we used the age-scaled scores of sub-tests. 
Table 1. The Average Score of Neuropsychological Tests in this Patients Group

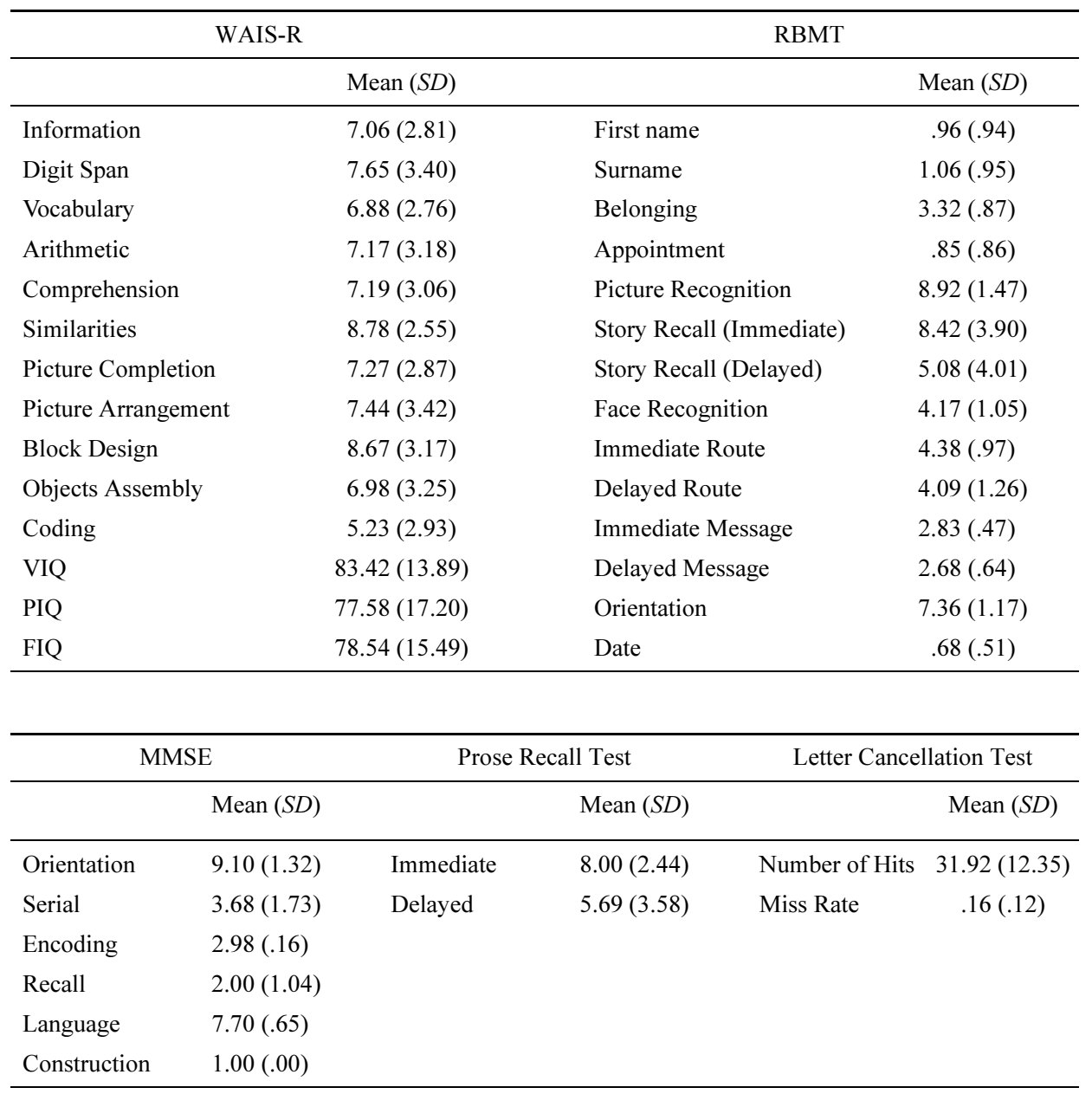

\section{RESULTS}

Table 2 showed the correlations between the subjective evaluation score of each cognitive status and its sub-tests. All of the sub-test scores of WAIS-R significantly correlated with the subjective evaluation score of scholastic ability. About half of the scores of the memory tests (i.e., RBMT and Prose Recall Test) also correlated with this subjective evaluation (e.g., Surname, and Appointment). Most of the sub-tests scores of RBMT correlated with the subjective evaluation of memory. Especially, Appointment, Story recall (delayed), Delayed Rote, and Delayed Message strongly correlated with the subjective evaluation of memory. Five sub-tests of RBMT also correlated with the subjective evaluation of attention (e.g., Immediate Rote, Delayed Route, and Orientation). Miss Rate of Letter Cancellation Test moderately correlated with attention. Finally, the 
Table 2. Pearson's Correlations Between Four Facets of Cognitive Status and Subtests

\begin{tabular}{|c|c|c|c|c|c|}
\hline & & $\begin{array}{c}\text { scholastic } \\
\text { ability }\end{array}$ & memory & attention & $\begin{array}{c}\text { processing } \\
\text { speed }\end{array}$ \\
\hline Information & WAIS-R & $.53 * *$ & .19 & .07 & .03 \\
\hline Digit Span & WAIS-R & $.29^{*}$ & .13 & .09 & .07 \\
\hline Vocabulary & WAIS-R & $.64 * *$ & $.29^{*}$ & .09 & .12 \\
\hline Arithmetic & WAIS-R & $.46^{* *}$ & .14 & .15 & .10 \\
\hline Comprehension & WAIS-R & $.49^{* *}$ & .05 & .08 & .08 \\
\hline Similarities & WAIS-R & $.55^{* *}$ & .25 & -.13 & .01 \\
\hline Picture Completion & WAIS-R & $.44 * *$ & .06 & -.09 & .16 \\
\hline Picture Arrangement & WAIS-R & $.41^{* *}$ & .16 & .17 & .18 \\
\hline Block Design & WAIS-R & $.43^{* *}$ & .19 & .23 & $.35^{*}$ \\
\hline Objects Assembly & WAIS-R & $.39 * *$ & .20 & .20 & .14 \\
\hline Coding & WAIS-R & $.48^{* *}$ & .25 & .22 & $.43 * *$ \\
\hline First name & RBMT & .22 & .21 & .12 & -.07 \\
\hline Surname & RBMT & $.33^{*}$ & $.31^{*}$ & .28 & .04 \\
\hline Belonging & RBMT & -.10 & $.35^{*}$ & .18 & -.17 \\
\hline Appointment & RBMT & $.32 *$ & $.50^{* *}$ & $.29^{*}$ & $.37 * *$ \\
\hline Picture Recognition & RBMT & -.02 & .24 & .02 & -.01 \\
\hline Story Recall (Immediate) & RBMT & $.57 * *$ & .28 & .08 & .16 \\
\hline Story Recall (Delayed) & RBMT & $.42 * *$ & $.49^{* *}$ & .26 & .17 \\
\hline Face Recognition & RBMT & .12 & .22 & -.15 & .10 \\
\hline Immediate Route & RBMT & .21 & $.38 * *$ & $.38 * *$ & .05 \\
\hline Delayed Route & RBMT & $.29 *$ & $.58 * *$ & $.41^{* *}$ & .10 \\
\hline Immediate Message & RBMT & .07 & .04 & .20 & -.11 \\
\hline Delayed Message & RBMT & .23 & $.40 * *$ & $.28^{*}$ & .10 \\
\hline Orientation & RBMT & .14 & $.55^{* *}$ & .14 & .04 \\
\hline Date & RBMT & $.30 *$ & $.31^{*}$ & $.44 * *$ & .26 \\
\hline Orientation & MMSE & $.42 *$ & $.51^{* *}$ & .19 & .29 \\
\hline Serial & MMSE & $.50 * *$ & .15 & .06 & .12 \\
\hline Encoding & MMSE & -.03 & .07 & .14 & .11 \\
\hline Recall & MMSE & -.01 & .10 & .07 & -.02 \\
\hline Language & MMSE & .28 & .11 & -.12 & -.12 \\
\hline Construction & MMSE & . & . & . & . \\
\hline Prose Recall (Immediate) & & $.58 * *$ & .26 & .12 & .06 \\
\hline Prose Recall (Delayed) & & $.48^{* *}$ & $.55^{* *}$ & .28 & .12 \\
\hline Letter Cancellation Test (Hit) & & $.33^{*}$ & .04 & .13 & .14 \\
\hline Letter Cancellation Test (Miss) & & -.08 & -.13 & $-.41 * *$ & -.06 \\
\hline
\end{tabular}

$* p<.05 \quad * * p<.01$

Note: All patients performed completely on Construction of MMSE (Max=1.0). Then, the Pearson's correlation between four facet of cognitive status and Construction could not be obtained. 
Table 3. Results of Stepwise Multi-regression Analysis

\begin{tabular}{ll}
\hline & Beta \\
\hline Scholastic ability & $.635^{* * *}$ \\
Vocabulary & \\
Memory & $.404^{* * *}$ \\
Delayed Route & $.308^{*}$ \\
Orientaiton (RBMT) & $.267^{*}$ \\
Appointment & $.369^{* * *}$ \\
Attention & $-.305^{*}$ \\
Date & \\
Miss Rate & $.429 * * *$ \\
Information Processing Speed & \\
Coding &
\end{tabular}

subjective evaluation of information processing speed correlated significantly with only Block Design (WAIS-R), Coding (WAIS-R), and Appointment (RBMT).

The goal of this study was to identify the useful sub-tests for a screening test battery. Then, we conducted stepwise multiple-regression analyses. The significance level was set at $p=.05$. Data were analyzed using by SPSS (10.0J) for Windows. Table 3 showed the results of the stepwise multiple-regression analyses.

For scholastic ability, a stepwise multiple-regression analysis indicated that Vocabulary (WAIS-R) was a powerful predictor $\left(R^{2}=.404, \mathrm{p}<.001\right)$.

For memory, a stepwise multiple-regression analysis revealed that Delayed Route (RBMT), Orientation (RBMT), and Appointment (RBMT) could predict memory function $\left(R^{2}=.538, p<.001\right)$.

For attention, a stepwise multiple-regression analysis showed that Date (RBMT) and Miss Rate (Letter Cancellation Test) could predict the attention status $\left(R^{2}=.284, p<.001\right)$.

For information processing speed, a stepwise multiple-regression analysis showed that Coding (WAIS-R) was a significant predictor $\left(R^{2}=.184, p<.001\right)$. In comparison with case of scholastic ability and memory, however, $R$ square was fairly low for both attention and information processing speed.

\section{DISCUSSION}

The multiple-regression analyses clarified the relationships between each criterion variable and patients' performance on the available neuropsychological tests. The findings of the present study were as follows: Vocabulary (WAIS-R) was the powerful 
predictor of scholastic ability. The predictive power of the other sub-tests of WAIS-R (e.g., Arithmetic) was weaker than that of Vocabulary, but these sub-tests highly correlated with scholastic ability. Some of the sub-tests of RBMT (i.e., Delayed route, Orientation and Appointment) could predict memory ability of patients. The predictive power of the other memory tests (e.g., Prose Recall) was weaker than that of these three memory tests. This might be due to that some aspects of memory functions were more selectively impaired after injury. Delayed Route (RBMT) can assess visuo-spatial memory, and Appointment (RBMT) is able to estimate prospective memory (Cockburn, et al., 1990). Recent studies suggested that visuo-spatial and prospective memories associate with executive function (Burgess, Quayle, \& Christopher, 2001; Palmer \& McDonald, 2000; Postle, Berger, Taich, \& D'Esposito, 2000). Memory functions depending on executive function might be selectively impaired after injury (McDowell, Whyte, \& D'Esposito, 1997).

Miss Rate of Letter Cancellation Test was a predictor of attention ability. However, a multiple-regression analysis showed that it could somehow predict the attention ability thought the $R$ square was moderately low. We hypothesized that Coding (WAIS-R) was the candidate for estimation of attention ability. Prigatano (1986) argued that Coding was useful to screen attention disorder, and Watanabe (1998) proposed that the score of Coding was frequently impaired after the injury. Consistent with their arguments, mean score of Coding of our patients was lower than that of normative population (see Table 1 and Shinagawa, et al., 1990). However, our results of multiple-regression analysis showed that Coding was not a powerful predictor for attention ability, suggesting that attention ability should be measured by the effect of cognitive load or distraction stimuli on the performance but not the performance itself in a trial (van Zomeren \& Brouwer, 1994). Finally, for information processing speed, a multiple-regression analysis showed that Coding was a significant predictor but the predictive power was fairly low. Inconsistent with our hypothesis, the performance on Letter Cancellation Test did not correlate with information processing speed.

As mentioned in the Introduction part, there is no appropriate screening test battery for TBI patient in Japan. MMSE has been frequently used for the elderly population in our country, and it might be regarded as a desirable screening test battery. However, the present study demonstrated that MMSE is not powerful enough to estimate cognitive status of TBI patients. Correlation analysis revealed that only Orientation and Serial correlated with scholastic ability, and Orientation correlated with memory moderately. Probably, MMSE might be too easy for our patients because they were relatively young ( $M=30.6$ years). Actually, their performance of Language and Construction nearly or completely approached the maximal level (Max score $=8.0$ or 1.0 , respectively). Though MMSE is suitable for elderly population, it must be inadequate as a screening test battery of TBI patients.

Based on the present findings, we would like to propose that Vocabulary (WAIS-R) and three sub-tests of RBMT (i.e., Delayed Route, Orientation and Appointment) would be candidates of the screening test for scholastic ability and memory, respectively. However, the results of multiple-regression analyses suggest that the available tests are 
not sufficient to estimate patient's attention and information processing speed. Recently, Hatta, Ito, and Yoshizaki (2001) developed Digit Cancellation Test (D-CAT) which focuses on the evaluation of attention and information processing speed. Therefore, the screening test battery which consists of Vocabulary (WAIS-R), Delayed route (RBMT), Orientation (RBMT), Appointment (RBMT), and D-CAT would be the best choice. The administration of this proposed battery takes about $20 \mathrm{~min}$. Of course, the screening test battery must be standardized by the appropriate test development technique in the further studies.

We believe that the results of the present study are suggestive. But we acknowledge some limitations of this study. For example, the number of patients was below what is needed to support the stepwise multiple-regression analysis. As mentioned early, this is due to the fact that clinicians have administered the available tests to TBI patients without deciding which tests address which aspects of cognitive functions. Accordingly identical test sets have not been administered to all patients for practical reasons (e.g., insufficient time for evaluation). Another problem is related to the fact that the criterion used was only the medical staffs' subjective evaluation. Use of other criteria such as injury severity might produce different results. But, in the present study, we could not obtain the full patients' medical information immediately after injury. Therefore, we chose to use only the medical staffs' subjective evaluation. A medical staff's subjective evaluation is very important because, by their final evaluation, patients are classified in the case of insurance, and a rehabilitation program and an employment guidance are decided. Therefore, we acknowledge that the results of this study should be interpreted with caution. At this point of time, however, the results of our study make lay the groundwork for the development of screening test battery. Limitations of this study involve the circumstances of Japanese medical practice (e.g., system of neuropsychological examination and the inter-relationship between the emergency and the rehabilitation hospitals). At present, it is difficult for us to eliminate them. However, systematic research of TBI patients just has started with support by Japanese Ministry of Health, Labour and Welfare. Therefore, these problems will be solved in the future researches.

\section{REFFERENCES}

Abe, J. 2000a. How to manage the disorder of behavior, psychiatry and mood? Sogo Rehabilitation, 28, $141-145$.

Abe, J. 2000b. How to manage TBI patients? Nursing Today, 15, 74-77.

Arciniegas, D., Olincy, A., Topkoff, J. McRae, K., Cawthra, E., Filley, C. M., Reite, M., \& Adler, L. E. 2000. Impaired auditory gating and P50 nonsuppression following traumatic brain injury. Journal of Neuropsychiatry \& Clinical Neurosciences, 12, 77-85.

Baddeley, A. D. 2000. The episodic buffer: A new component of working memory. Trends in Cognitive Sciences, 4, 417-423.

Baddeley, A. D., \& Hitch, G. J. 1974. Working memory. In G. H. Bower (Ed.), The psychology of learning and motivation: Advances in research and theory, Vol. 8 (pp. 47-89). New York: Academic Press.

Burgess, P. W., Quayle, A., \& Christopher, D. 2001. Brain regions involved in prospective memory as determined by positron emission tomography. Neuropsychologia, 39, 545-555.

Chan, R. C. 2001. A further study on the sustained attention response to task (SART): The effect of age, 
gender and education. Brain Injury, 15, 819-829.

Christensen, A. 1975. Luria's neuropsychological investigation: Text. New York: Spectrum Publications.

Christodoulou, C., DeLuca, J., Ricker, J. H., Madigan, N. K., Bly, B. M., Lange, G., Kalnin, A. J., Liu, W. C., Steffener, J., Diamond, B. J., \& Ni, A. C. 2001. Functional magnetic resonance imaging of working memory impairment after traumatic brain injury. Journal of Neurology, Neurosurgery \& Psychiatry, 71, 161-168.

Cockburn, J., Wilson, B., Baddeley, A., \& Hiorns, R. 1990. Assessing everyday memory in patients with dysphasia. British Journal of Clinical Psychology, 29, 353-360.

Cooke, D. L., \& Kausler, D. H. 1995. Content memory and temporal memory for actions in survivors of traumatic brain injury. Journal of Clinical \& Experimental Neuropsychology, 17, 90-99.

Diller, L., \& Weinberg, J. 1977. Hemi-inattention in rehabilitation: The evolution of a rational remediation program. Advances in Neurology, 18, 63-82.

Ewing-Cobbs, L., Fletcher, J. M., Levin, H. S., Iovino, I., \& Miner, M. E. 1998. Academic achievement and academic placement following traumatic brain injury in children and adolescents: A two-year longitudinal study. Journal of Clinical \& Experimental Neuropsychology, 20, 769-781.

Fenwick, T., \& Anderson, V. 1999. Impairments of attention following childhood traumatic brain injury. Child Neuropsychology, 5, 213-223.

Folstein, M. F., Folstein, S. E., \& McHugh, P. R. 1975. "Mini-mental state". A practical method for grading the cognitive state of patients for the clinician. Journal of Psychiatric Research, 12, 189-198.

Hatta, T., Ito, Y., \& Yoshizaki, K. 2001. Digit Cancellation Test (D-CAT). Osaka: Union Press.

Jennett, B., \& Teasdale, G. 1977. Aspects of coma after severe head injury. Lancet, 23, 878-881.

Lezak, M. D. 1976. Neuropsychological assessment. New York: Oxford University Press.

Leclercq, M., Couillet, J., Azouvi, P., Marliner, N., Martin, Y., Strypstein, E., \& Rousseaux, M. 2000. Dual task performance after severe diffuse traumatic brain injury or vascular prefrontal damage. Journal of Clinical \& Experimental Neuropsychology, 22, 339-350.

Milberg, W. 1996. Issues in the assessment of cognitive function in dementia. Brain \& Cognition, 31, 114-132.

McDowell, S., Whyte, J., \& D’Esposito, M. 1997. Working memory impairments in traumatic brain injury: Evidence from a dual-task paradigm. Neuropsychologia, 35, 1341-1353.

Palmer, H. M., \& McDonald, S. 2000. The role of frontal and temporal lobe processes in prospective remembering. Brain \& Cognition, 44, 103-107.

Ponsford, J., Sloan, S., \& Snow, P. 1995. Traumatic brain injury. Hove: Psychology Press.

Postle, B. R., Berger, J. S., Taich, A. M., \& D’Esposito, M. 2000. Activity in human frontal cortex associated with spatial working memory and saccadic behavior. Journal of Cognitive Neuroscience, 12, 2-14.

Prigatano, G. P. 1986. Neuropsychological rehabilitation after brain injury. Baltimore: Johns Hopkins University Press.

Rose, D., \& Johnson, D. A. 1996. Brain injury and after: Towards improved outcome. New York: Wiley.

Shum, D. H. K., Harris, D., \& Gorman, J. G. 2000. Effects of severe traumatic brain injury on visual memory. Journal of Clinical \& Experimental Neuropsychology, 22, 25-39.

Shum, D. H. K., Jamieson, E., Bahr, M., \& Wallace, G. 1999. Implicit and explicit memory in children with traumatic brain injury. Journal of Clinical \& Experimental Neuropsychology, 21, 149-158.

Shum, D. H. K., Valentine, M., \& Cutmore, T. 1999. Performance of individuals with severe long-term traumatic brain injury on time-, event-, and activity-based prospective memory tasks. Journal of Clinical \& Experimental Neuropsychology, 21, 49-58.

Shinagawa, F., Kobayashi, S., Fujita, K., \& Maekawa, H. 1990. Manual for the Japanese Wechsler Adult Intelligence Scale-Revised. Tokyo: Nihon Bunka Kagakusha Corporation.

Sohlberg, M. M., \& Mateer, C. A. 1989. Introduction to cognitive rehabilitation: Theory and practice. New York: Guilford Press.

Tate, R. L. 1999. Executive dysfunction and characterological changes after traumatic brain injury: Two side of the same coin? Cortex, 35, 39-55.

Van Zomeren, A. H., \& Brouwer, W. H. 1994. Clinical neuropsychology of atttention. New York: Oxford University Press.

Watanabe, O. 1998. Cognitive disorders. Journal of Clinical Rehabilitation, 7, 138-144.

Whyte, J., Schuster, K., Potansky, M., Adams, J., \& Coslett, H. B. 2000. Frequency and duration of inattentive behavior after traumatic brain injury: Effects of distraction, task and practice. Journal of the 
International Neuropsychological Society, 6, 1-11.

Wechsler, D. 1981. Manual for the Wechsler Adult Intelligence Scale-Revised. New York: The psychological Corporation.

(Manuscript received March 19, 2003; Revision accepted September 11, 2003) 